\title{
A passively mode-locked Holmium fiber oscillator based on a Nonlinear Amplifying Loop Mirror operating at 2050 nm
}

\author{
Christoph Mahnke ${ }^{1}$, Yuxuan Ma ${ }^{1}$, Sarper Salman ${ }^{1,2}$, Christoph M. Heyl ${ }^{1,2}$ and Ingmar Hartl ${ }^{1}$ \\ 1. Deutsches Elektronen-Synchrotron DESY, Notkestraße 85, 22607 Hamburg, Germany \\ 2. Helmholtz-Institute Jena, Fröbelstieg 3, 07743 Jena, Germany \\ christoph.mahnke@desy.de
}

In recent years, mode-locked fiber lasers have proven to be reliable sources for femto- and picosecond pulses for various applications like communications, metrology or biomedical applications [1]. We present first results on a Ho:fiber oscillator based on a Nonlinear Amplifying Loop Mirror (NALM) configuration, intended for seeding a Ho:YLF regenerative amplifier [2]. The NALM technique, when using polarization-maintaining (PM) fibers, allows self-starting, low noise, alignment-free, robust long term stable operation [1]. It is implemented here - to the best of our knowledge- for the first time using a Ho-fiber without Tm co-doping.
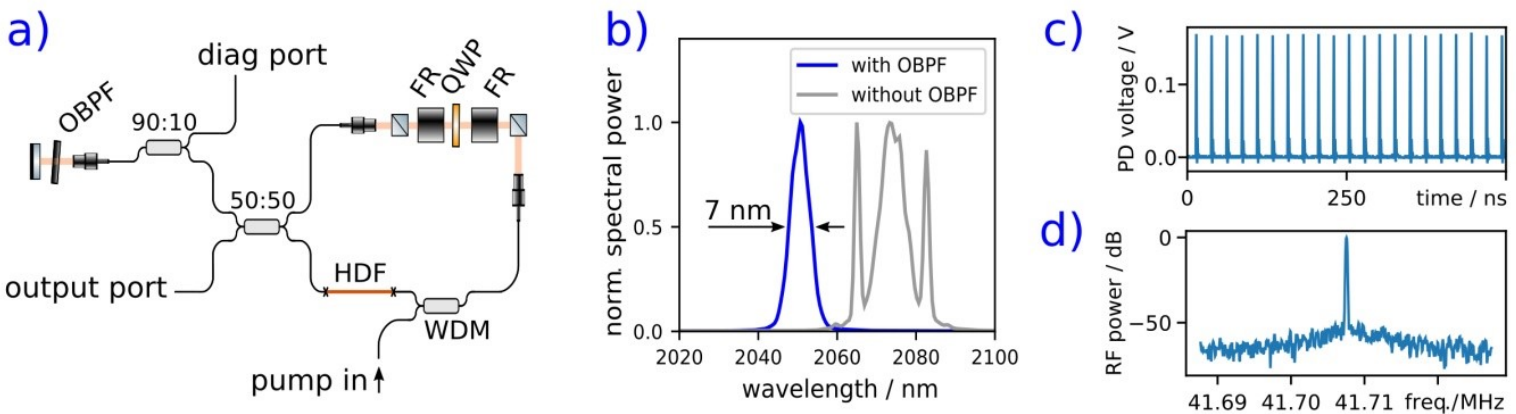

Fig.1: a) overview of the setup. b) optical spectra of mode-locked operation. c) pulse train in the time domain. d) radio-frequency spectrum (resolution bandwidth $100 \mathrm{~Hz}$ ) around the repetition rate.

The schematic setup of the oscillator is shown in figure 1 a). It consists of a Nonlinear Amplifying Loop Mirror, a linear arm and a rejection port which are connected by a 50:50 fiber coupler. All passive fiber sections are PM fiber optimized for 2 um applications (Nufern PM1950).

The NALM loop is formed by a section of PM Holmium-doped gain fiber (Nufern PM-HDF-10/130-15FA) and additional sections of passive fiber. The asymmetric placement of the active fiber in the loop provides the difference in nonlinear phase shifts for clockwise and counter-clockwise propagation that is needed to introduce an intensity-dependent reflectivity of the NALM. An additional linear nonreciprocal phase shift is introduced in a short free space section with two Faraday rotators (FR) and a quarter wave plate (QWP), enabling self-starting mode-locking $[3,4]$.

The linear arm of the oscillator contains a 10:90 coupler used for diagnostics and a free space-section with a small metallic end mirror mounted on a piezo actuator intended for cavity length stabilization. An optical bandpass filter (OBPF) can be placed in the free space section to select the operation wavelength. To pump the system, light from a commercial Thulium pump laser is coupled into the oscillator using a wavelength-division multiplexer (WDM).

Mode-locking of the oscillator is initiated when the power of the pump light is set to about $1.4 \mathrm{~W}$. Typically, the laser starts pulsing in a multi-pulse modus. A stable, single pulse regime is reached when the pump power is reduced down to about $700 \mathrm{~mW}$; mode-locking is lost at about $580 \mathrm{~mW}$ of pump power.

Without the OBPF in the linear arm, the oscillator shows a typical soliton spectrum with the characteristic Kelly sidebands (gray spectrum in Fig. 1b)). Placing and tilting the OBPF allows to adjust the wavelength to about $2050 \mathrm{~nm}$ (blue spectrum in Fig. 1b)), which is required for the intended seeding of a Ho:YLF amplifier. The OBPF greatly suppresses the Kelly sidebands. The bandwidth of the optical spectrum is about $7 \mathrm{~nm}$, supporting pulse durations of about 1 ps. The repetition rate is set to $41.7 \mathrm{MHz}$ and will be stabilized in the future using the piezo-actuated end mirror. Fig. 1c) shows the temporal trace of the pulses generated, recorded with a fast photo diode. The radio frequency spectrum in the vicinity of the repetition rate is depicted in Fig. 1d).

The output power from the output port is $4 \mathrm{~mW}$, corresponding to a pulse energy of about 95 pJ. About 2 $\mathrm{mW}$ of power from the coupler in the linear arm is available for diagnostics.

\section{References}

[1] J. Kim and Y. Song, "Ultralow-noise mode-locked fiber lasers and frequency combs: principles, status, and applications," Adv. Opt. Photonics 8, 465 (2016).

[2] P. Kroetz, et al. "Study on laser characteristics of Ho: YLF regenerative amplifiers: Operation regimes, gain dynamics, and highly stable operation points," Appl. Phys.B, 123, 126 (2017).

[3] N. Kuse, et al. "All polarization-maintaining Er fiber-based optical frequency combs with nonlinear amplifying loop mirror," Opt. Express 24, 3095 (2016).

[4] W. Hänsel et al., "All polarization-maintaining fiber laser architecture for robust femtosecond pulse generation," Appl. Phys. B 123, 41 (2017). 